\title{
Spin and charge signatures of topological superconductivity in Rashba nanowires
}

\author{
Paweł Szumniak, ${ }^{1,2}$ Denis Chevallier, ${ }^{1}$ Daniel Loss, ${ }^{1}$ and Jelena Klinovaja ${ }^{1}$ \\ ${ }^{1}$ Department of Physics, University of Basel, Klingelbergstrasse 82, 4056 Basel, Switzerland \\ ${ }^{2}$ AGH University of Science and Technology, Faculty of Physics and Applied Computer Science, al. Mickiewicza 30, 30-059 Kraków, Poland
}

(Received 8 March 2017; published 5 July 2017)

\begin{abstract}
We consider a Rashba nanowire with a proximity gap which can be brought into the topological phase by tuning external magnetic field or chemical potential. We study spin and charge of the bulk quasiparticle states when passing through the topological transition for open and closed systems. We show, analytically and numerically, that the spin of bulk states around the topological gap reverses its sign when crossing the transition due to band inversion, independent of the presence of Majorana fermions in the system. This spin reversal can be considered as a bulk signature of topological superconductivity that can be accessed experimentally. We find a similar behavior for the charge of the bulk quasiparticle states, also exhibiting a sign reversal at the transition. We show that these signatures are robust against random static disorder.
\end{abstract}

DOI: 10.1103/PhysRevB.96.041401

Introduction. Topological phases of condensed matter systems [1,2] have attracted a lot of attention over many years due to their high promise for applications such as topological quantum computation $[3,4]$. One of the hallmarks of such phases, in particular of topological superconductivity, are zero-energy modes such as Majorana fermions (MF) that emerge at the edges of the system. Various candidate materials can host such topological states [5-21] but one of the most promising platforms are semiconducting nanowires of InAs or InSb material, with strong Rashba spin orbit interaction (SOI), subjected to an external magnetic field and in proximity to an $s$-wave superconductor $[22,23]$. Experimental evidence has been reported for topological phases in such wires [24-31] as well as in magnetic atomic chains on superconducting substrates [32-34]. However, most of the work so far has focused on the detection of the MFs in these nanowires and not on their bulk properties. This is quite surprising given the fact that the unambiguous identification of MFs from transport data alone can be challenging [35-43]. It is thus of great interest to look for alternative signatures of topological phases and to address the question how the bulk states change when passing from trivial to topological phase and if these changes appear in physically observable quantities.

In this work, we show that the phase of a topological superconductor can be monitored by bulk states, in particular by certain spin and charge degrees of freedom. Quite remarkably, we find that the sign of the spin component along the magnetic field reverses for low-momentum states close to the Fermi level when the system passes through the phase transition, and similarly for the charge of such bulk states. This sign reversal is a direct consequence of the band inversion at the transition point and is directly accessible by spin- and energy resolved measurements. Another remarkable feature is that this signature is independent of boundary effects and thus unrelated to the presence of MFs. To demonstrate these findings, we perform analytical and numerical calculations for both closed and open systems and for various parameter regimes which are relevant for InAs or InSb nanowires used in recent experiments [24-31]. We also demonstrate that these effects are robust against static random disorder.
Model. We consider a one-dimensional Rashba nanowire aligned along the $x$-axis and placed on top of an $s$-wave superconductor in the presence of an external magnetic field applied along the nanowire axis (see Fig. 1). The system can be modeled by the tight-binding Hamiltonian

$$
\begin{aligned}
H= & \sum_{j=1}^{N-1}\left[\Psi_{j+1}^{\dagger}\left(-t-i \tilde{\alpha} \sigma_{y}\right) \tau_{z} \Psi_{j}+\text { H.c. }\right] \\
& +\sum_{j=1}^{N} \Psi_{j}^{\dagger}\left[(2 t-\mu) \tau_{z}+\Delta_{\mathrm{sc}} \tau_{x}+\Delta_{Z} \sigma_{x}\right] \Psi_{j},
\end{aligned}
$$

where $\Psi_{j}=\left(c_{j \uparrow}, c_{j \downarrow}, c_{j \downarrow}^{\dagger},-c_{j \uparrow}^{\dagger}\right)^{T}$ is given in standard Nambu representation. The creation operator $c_{j \sigma}^{\dagger}$ acts on an electron with spin $\sigma$ located at site $j$ in a chain of $N$ sites with lattice constant $a$. The Zeeman splitting $2 \Delta_{Z}=g \mu_{B} B_{x}$ is determined by the $g$ factor and by the strength of the external magnetic field $B_{x}$. The superconducting pairing term $\Delta_{\mathrm{sc}}$ is induced in the nanowire via proximity effect by the $s$-wave superconductor. The chemical potential of the nanowire $\mu$ is calculated from the SOI energy and $t$ is the hopping amplitude. The Pauli matrices $\sigma_{i}\left(\tau_{i}\right)$ act on spin (particle-hole) space and $\tilde{\alpha}$ is the spin-flip hopping amplitude used to model the Rashba SOI. By diagonalizing numerically $H$, we find the spectrum $E_{n}$ and corresponding wave functions $\Phi_{n}(j)$ labeled by the index $n=1, \ldots, 4 N$.

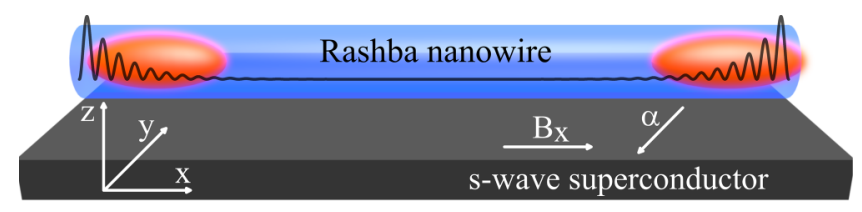

FIG. 1. A semiconducting nanowire proximity-coupled to an $s$-wave superconductor. The Rashba SOI vector $\alpha$ points in the $y$ direction and an external magnetic field $B_{x}$ is applied in the $x$ direction. In the topological phase, spin, and chargeless MFs (orange ovals) are localized at the ends, with corresponding probability density indicated by black lines. 


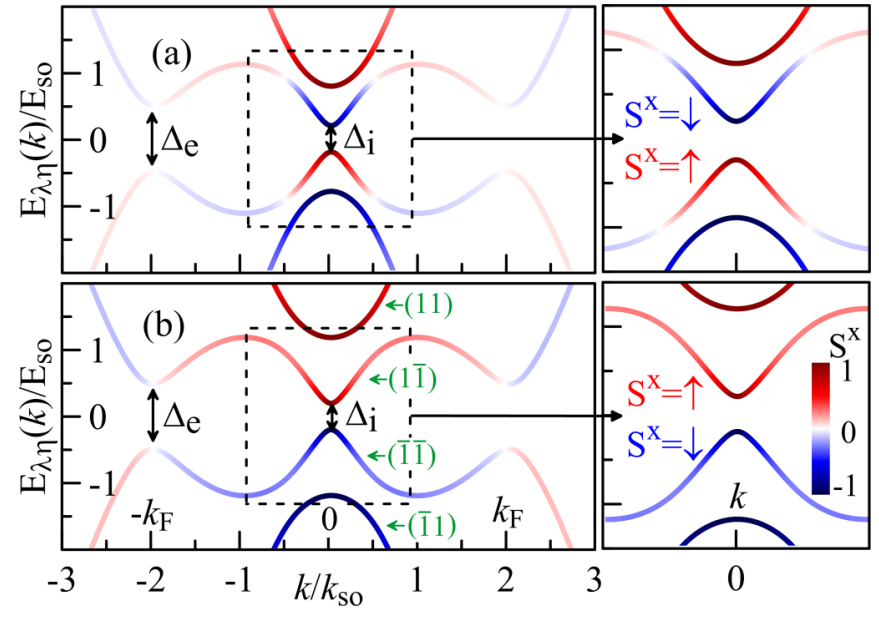

FIG. 2. The spectrum of Eq. (3) in the trivial (a) and topological (b) phases for closed systems. The blue/red color of dispersion lines $E_{\lambda \eta}(k)$ indicates the negative/positive sign of the spin component $S_{\lambda \eta}^{x}(k)$ for states of a given $\lambda \eta$ band. Around $k=0$, the $\operatorname{spin} S_{\lambda \overline{1}}^{x}(k)$ of states with energies closest to the Fermi level $\mu=0$ in the trivial phase is opposite to the one in the topological phase. The insets show the spectrum around $k=0$ where such a sign reversal of $S_{\lambda \overline{1}}^{x}(k)$ occurs. We used the following parameters: $\mu=0, \Delta_{\mathrm{sc}}=0.5 E_{\mathrm{so}}$, and $\Delta_{Z}=0.3 E_{\mathrm{so}}\left(\Delta_{Z}=0.7 E_{\mathrm{so}}\right)$ in the trivial (topological) phase, so that the interior gap $\Delta_{i}=2\left|\Delta_{Z}-\Delta_{\text {sc }}\right|$ remains the same.

In order to study analytically the bulk states of the system, we also write $H$ in momentum space. By imposing periodic boundary conditions, we can introduce the momentum $k$, $c_{j \sigma}=\sum_{j} c_{k \sigma} e^{-i j k a} / \sqrt{N}$, and $\Psi_{k}=\left(c_{k \uparrow}, c_{k \downarrow}, c_{-k \downarrow}^{\dagger},-c_{-k \uparrow}^{\dagger}\right)^{T}$. We then obtain $H=\sum_{k} \Psi_{k}^{\dagger} \mathcal{H}(k) \Psi_{k}$ with

$$
\begin{aligned}
\mathcal{H}(k)= & {\left[2 t-2 t \cos (k a)-\mu+2 \tilde{\alpha} \sin (k a) \sigma_{y}\right] \tau_{z} } \\
& +\Delta_{\mathrm{sc}} \tau_{x}+\Delta_{Z} \sigma_{x} .
\end{aligned}
$$

In the continuum limit $k a \ll 1$ [45], we get

$$
\mathcal{H}(k)=\left(\frac{\hbar^{2} k^{2}}{2 m}-\mu+\alpha k \sigma_{y}\right) \tau_{z}+\Delta_{\mathrm{sc}} \tau_{x}+\Delta_{Z} \sigma_{x} .
$$

The correspondence between the tight-binding and continuum model is then given by $t=\hbar^{2} /\left(2 m a^{2}\right)$, where $m$ is an effective electron mass [40]. The spin-flip hopping amplitude $\tilde{\alpha}$ is related to the SOI strength by $\tilde{\alpha}=\alpha / 2 a$. The corresponding SOI energy (momentum) is defined as $E_{\mathrm{so}}=\tilde{\alpha}^{2} / t \equiv m \alpha^{2} / 2 \hbar^{2}$ $\left(k_{\mathrm{so}}=m \alpha / \hbar^{2}\right)$. By diagonalizing $\mathcal{H}(k)$ [see Eq. (2) or (3)], we arrive at analytical expressions for the eigenvalues $E_{\lambda \eta}(k)$ and corresponding eigenstates $\Phi_{\lambda \eta}(k)$ (see Ref. [44]). In total, there are four bands, labeled by $\lambda$ and $\eta$, where $\lambda=1(\lambda=\overline{1})$ labels bands with positive (negative) energy and $\eta=\overline{1}$ the bands closest to the Fermi level, see Fig. 2.

The lowest band $\eta=\overline{1}$ has gaps at $k=0$, which we call the interior gap $\Delta_{i}=2\left|\Delta_{z}-\sqrt{\mu^{2}+\Delta_{\text {sc }}^{2}}\right|$, and at finite Fermi points $k= \pm k_{F}$, which we call the exterior gap $\Delta_{e}=2\left|E_{\lambda \overline{1}}\left(k_{F}\right)\right|$. The central quantities of interest are the spin $\mathbf{S}_{n}\left[\mathbf{S}_{\lambda \eta}(k)\right]$ and the charge $Q_{n}\left[Q_{\lambda \eta}(k)\right]$ of the bulk quasiparticles states at given energy $E_{n}\left[E_{\lambda \eta}(k)\right]$, defined in the tight-binding (continuum) model as

$$
\begin{gathered}
\mathbf{S}_{n}=\sum_{j=1}^{N} \Phi_{n}^{\dagger}(j) \sigma \Phi_{n}(j), \\
\mathbf{S}_{\lambda \eta}(k)=\Phi_{\lambda \eta}^{\dagger}(k) \sigma \Phi_{\lambda \eta}(k), \\
Q_{n}=-\sum_{j=1}^{N} \Phi_{n}^{\dagger}(j) \tau_{z} \Phi_{n}(j), \\
Q_{\lambda \eta}(k)=-\Phi_{\lambda \eta}^{\dagger}(k) \tau_{z} \Phi_{\lambda \eta}(k) .
\end{gathered}
$$

Here, the spin and charge are measured in units of $\hbar / 2$ and electron charge $|e|$, respectively. We note that in contrast to previous works [51,52], our definitions of spin and charge involve all four components of the wave function. Due to the periodic boundary conditions the system describes a closed ring and no MF can occur (for open systems, see below).

Spin and charge inversion at the topological phase transition. We focus now on the spin and charge of bulk states of the nanowire in the trivial $\left(\Delta_{Z}^{2}<\mu^{2}+\Delta_{\mathrm{sc}}^{2}\right)$ and in the topological $\left(\Delta_{Z}^{2}>\mu^{2}+\Delta_{\mathrm{sc}}^{2}\right)$ phases, see Fig. 2. The most interesting behavior occurs close to $k=0$, where the topological phase transition takes place as $\Delta_{i}=0$ for the $\eta=\overline{1}$ band. Quite remarkably, we observe a sign reversal of the spin component along the magnetic field, $S_{\lambda \overline{1}}^{x}(k)$, when the system is tuned from trivial to topological phase. In Fig. 2(a), the system is shown in the trivial phase where $S_{1 \overline{1}}^{x}(k)\left[S_{\overline{1} \overline{1}}^{x}(k)\right]$ around $k=0$ is negative (positive) for the electron (hole) $\eta=\overline{1}$ band, while the sign reverses when the system is tuned into the topological phase by changing the magnetic field, see Fig. 2(b). This change of sign is a direct consequence of the band inversion associated with the topological phase transition. Consequently, by measuring the spin component $S^{x}$ along the field $B_{x}$, one can determine whether the system is in the topological or trivial phase, even in the absence of any MFs. This finding opens up new experimental perspectives to identify topological superconductivity by measuring bulk state properties close to the Fermi level.

We note that there is also a residual spin component along the SOI axis $S^{y}(k)$, the sign of which, however, is the same both in the topological and trivial phase and thus cannot be used to distinguish phases. Moreover, due to the symmetry of the system, the spin projection $S^{z}(k)$ is always zero. In Ref. [44], we provide the analysis of all spin components $S^{i}(k)$ as a function of momentum $k$. We finally note that similar behavior as for spin is found also for the quasiparticle charge as shown in Ref. [44]. Indeed, close to $k=0$ and for the negative value of $\mu$ in the topological (trivial) phase, $Q_{1 \overline{1}}(k)$ is positive (negative) while $Q_{\overline{1} \overline{1}}(k)$ is negative (positive). For positive values of $\mu$, the situation is opposite. Again, this sign reversal can be used as a detection tool for topological superconductivity, independent of MFs. In Ref. [44], we also demonstrate that our results hold for nanowires with several subbands.

Phase diagram. To test if the spin $S_{\lambda \overline{1}}^{x}$ and charge $Q_{\lambda \overline{1}}$ of the bulk states with momentum close to $k=0$ allows one to distinguish reliably between trivial and topological phase, we determine the phase diagram as a function of magnetic field $\Delta_{Z}$ and chemical potential $\mu$ at fixed momentum, see Fig. 3, again 


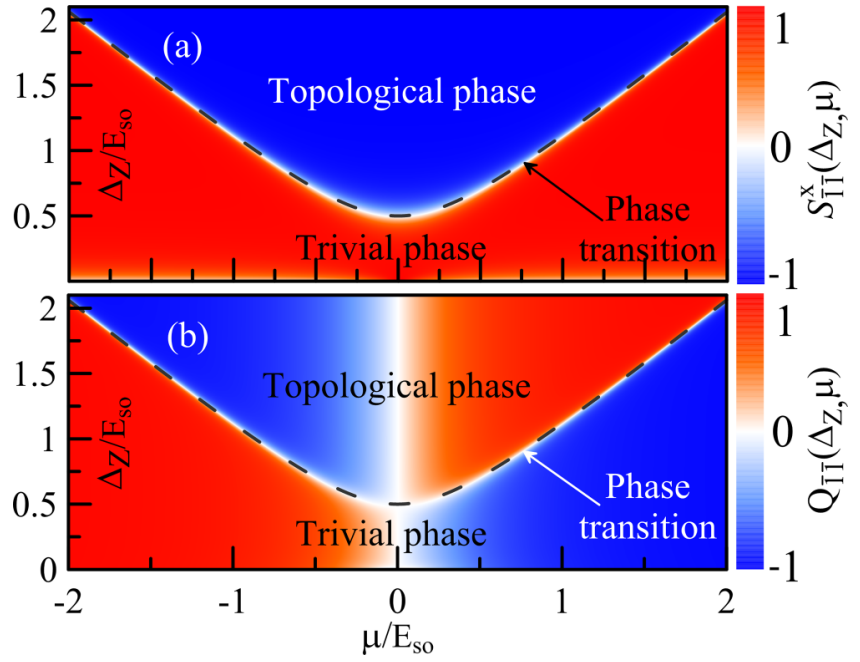

FIG. 3. Phase diagram as function of chemical potential $\mu$ and magnetic field $\Delta_{Z}$ by measuring (a) the $x$ component of the quasiparticle spin $S_{\overline{1} \overline{1}}^{x}$ and (b) the quasiparticle charge $Q_{\overline{1} \overline{1}}$ at fixed momentum $k=0.05 k_{\mathrm{so}}$ and fixed superconducting pairing $\Delta_{\mathrm{sc}}=0.5 E_{\mathrm{so}}$. Indeed, the phase boundary at the topological transition line $\Delta_{Z}=\sqrt{\Delta_{\mathrm{sc}}^{2}+\mu^{2}}$ (dashed line) is very well visible. A similar phase diagram is found for the states above $\mu\left(S_{1 \overline{1}}^{x}\right.$ and $\left.Q_{1 \overline{1}}\right)$, differing only in a global minus sign due to particle-hole symmetry.

for the closed system without MFs. The results are obtained for the bulk states from the $\overline{1} \overline{1}$ band, using Eq. (3). The phase transition at $\Delta_{Z}^{2}=\Delta_{\mathrm{sc}}^{2}+\mu^{2}$ is clearly indicated by the reversal of signs of both the spin $S_{\lambda \overline{1}}^{x}$ and charge $Q_{\lambda \overline{1}}$. Moreover, the boundary separating the two phases is sharp. We note that the charge reverses its sign at $\mu=0$. In contrast to the topological phase transition, this phase boundary is smooth.

The analytical expressions for charge and spin of the bulk states, obtained from Eqs. (5) and (7), are too lengthy to be shown here. However, since we are mainly interested in the features around $k=0$, we can expand these formulas for small momenta away from the phase transition (for simplicity, we also put $\mu=0$ ). For the $\lambda \overline{1}$ bands, we get in leading order,

$$
\begin{gathered}
S_{\lambda \overline{1}}^{x}(k)=\lambda \operatorname{sign}\left(\Delta_{Z}-\Delta_{\mathrm{sc}}\right)\left[1-\frac{(\alpha k)^{2}}{2\left(\Delta_{Z}-\Delta_{\mathrm{sc}}\right)^{2}}\right], \\
Q_{\lambda \overline{1}}(k)=\lambda \operatorname{sign}\left(\Delta_{Z}-\Delta_{\mathrm{sc}}\right) \frac{\hbar^{2} k^{2}}{2 m \Delta_{\mathrm{sc}}} .
\end{gathered}
$$

We can clearly see that around $k=0$ the sign of $S_{\lambda \overline{1}}^{x}$ is proportional to the sign of the topological gap $\Delta_{Z}-\Delta_{\mathrm{sc}}$. Thus one can consider $S_{\lambda \overline{1}}^{x}(k)$ as an order parameter that distinguishes between topological and trivial phases. One also notices that the sign of the quasiparticle charge is proportional to the sign of the topological gap, however, it changes only quadratically in $k$.

So far, we have studied systems with strong SOI, which is generally believed to be the case for InSb or InAs nanowire [24]. However, the sharpness of the boundary between two phases determined by $S_{\lambda \overline{1}}^{x}$ depends on the strength of the SOI
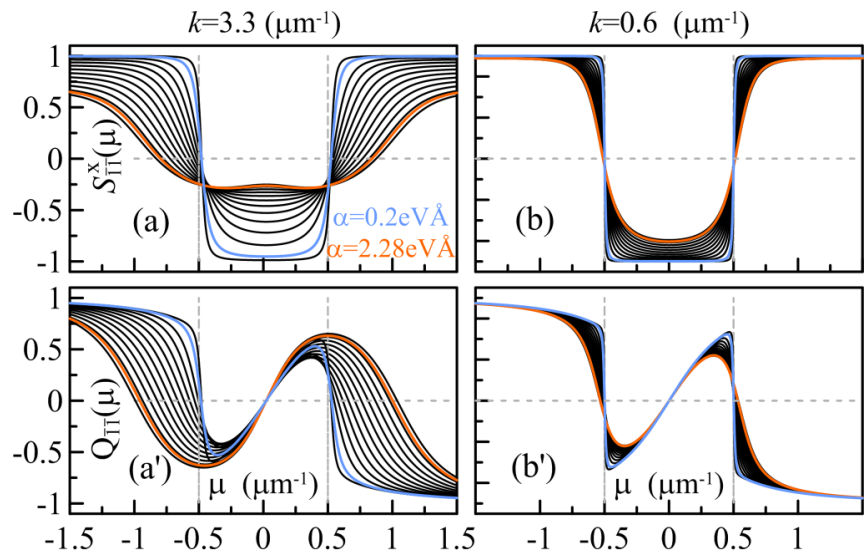

FIG. 4. Spin projection $S_{\overline{1} \overline{1}}^{x}\left[(\mathrm{a})\right.$ and (b)] and charge $Q_{\overline{1} \overline{1}}$ $\left[\left(a^{\prime}\right)\right.$ and $\left.\left(b^{\prime}\right)\right]$ of bulk states as a function of the chemical potential $\mu$ for two values of momenta [(a) and (a')] $k=3.3 \mu \mathrm{m}^{-1}$ and [(b) and (b')] $k=0.6 \mu \mathrm{m}^{-1}$ for various values of the SOI strength: $\alpha=0.1,0.2,0.4, \ldots, 2.2,2.28,2.4 \mathrm{eV} \AA$. The parameters of the system are $\Delta_{\mathrm{sc}}=0.5 \mathrm{meV}$ and $\Delta_{Z}=0.7 \mathrm{meV}$. The sign of charge and spin reverses as the system undergoes the topological phase transition at $\mu= \pm 0.5 \mathrm{meV}$ (denoted by gray dashed vertical lines). The boundary between phases is most pronounced for the states close to $k=0$ and almost independent of the SOI.

as seen from Eq. (8). To understand this dependence better, we study the evolution of $S_{\lambda \overline{1}}^{x}$ and $Q_{\lambda \overline{1}}$ as a function of $\mu$ for two different values of $k$, see Fig. 4 . Generally, we observe that the boundary between the topological and trivial phase is sharper for small values of $k$ and almost insensitive to the SOI strength. To conclude, the reversal of the sign of $Q_{\lambda \overline{1}}$ and $S_{\lambda \overline{1}}^{x}$ is well visible at small $k$ for all values of SOI.

Open systems with MFs. So far, we have considered closed systems not supporting MFs. In realistic setups, nanowires are open with finite length and momentum is not a good quantum number. In addition, finite systems in the topological phase host MFs at the wire ends and it is apriori not clear if their presence does not mask the signatures of topological phase transition found for closed systems above. Thus we focus now on such finite systems using realistic parameters [24,46]. First, we compare results obtained from Eq. (2) for closed systems with periodic boundary conditions with the ones obtained from tight-binding calculations using Eq. (1) for open systems with vanishing boundary conditions, see Fig. 5. Our numerical simulations give excellent agreement between the two models for parameters for which $\Delta_{i}<\Delta_{e}$. In the opposite regime, the exterior and interior branches are mixed, thus, one needs to involve momentum-resolved measurements. Thus, the spin component $S^{x}$ and its reversal can serve as a detection tool also in open systems. We obtain similar results also for the quasiparticle charge $Q_{n}$, see Ref. [44]. All this confirms that the topological phase transition of the bulk can be detected in the same way. The sign reversal of spin and charge does not depend on boundaries of the system, and is thus independent of the presence of MFs. This provides an advantage over detecting the topological phase via the presence of MFs, which could either leak into the lead [45] or be masked by disorder effects [37-40]. To show that our results are robust against disorder, we add random on-site fluctuations to $\mu$, 


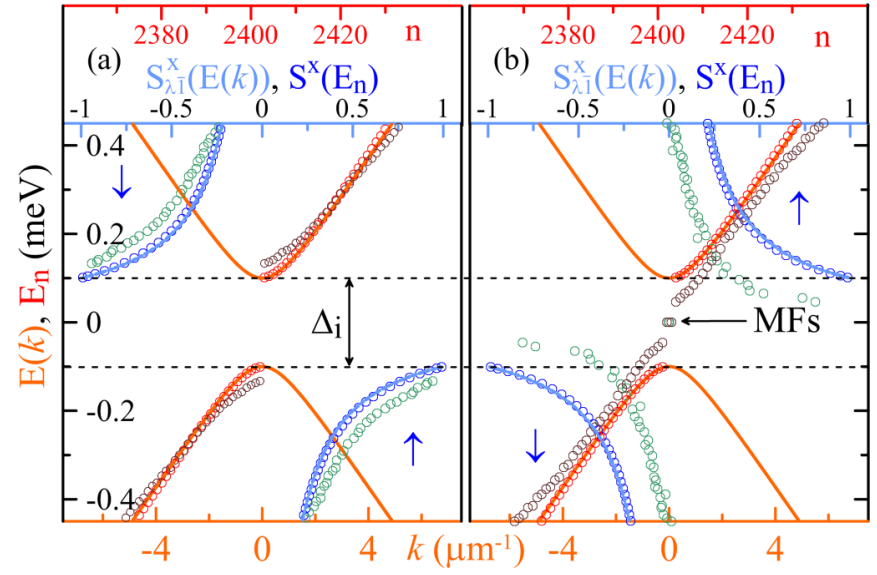

FIG. 5. The energy spectrum and spin $S^{x}$ in the trivial (a) and topological (b) phase for open and closed systems. We find excellent agreement between $E_{n}$ (red circles) and $S^{x}\left(E_{n}\right)=S_{n}^{x}$ (blue circles) obtained from the tight-binding model for open systems supporting MFs [see Eq. (1)] and $E_{\lambda \overline{1}}(k)$ (orange solid line) and $S_{\lambda \overline{1}}^{x}(k)$ obtained for closed systems without MFs [see Eq. (2)]. The topological phase transition is clearly indicated by the sign reversal of $S^{x}$ also in open systems. The trivial phase is plotted for $\Delta_{Z}=0.4 \mathrm{meV}$ while the topological one for $\Delta_{Z}=0.6 \mathrm{meV}$. The other parameters are fixed as $\Delta_{\mathrm{sc}}=0.5 \mathrm{meV}, \mu=0, \alpha=0.9 \mathrm{eV} \AA\left(E_{\mathrm{so}} \approx 1 \mathrm{meV}\right), N=1200$, and $m=0.015 m_{e}$, with $m_{e}$ being the bare electron mass, for InSb nanowires $(t=10 \mathrm{meV}, a=15 \mathrm{~nm})$. In addition, we consider a random on-site disorder potential of strength $\left|\delta \mu_{j}\right|<1 \mathrm{meV}$. The spin $S^{x}\left(E_{n}\right)$ (green circles) corresponding to $E_{n}$ (brown circles) of the disordered nanowire undergoes the same reversal of sign as in the clean case, demonstrating its robustness. i.e. set $\mu_{i}=\mu+\delta \mu_{i}$ in Eq. (1). We find that even for disorder strengths exceeding the proximity gap $\Delta_{\mathrm{sc}}$, the reversal of sign in spin is hardly affected, see Fig. 5. In Ref. [44], we study effects of static potential and magnetic disorder on the charge and spin signature in more detail. Again, we conclude that the proposed signature could be used to characterize the topological phase transition.

Conclusions. We have shown that the topological phase transition in Rashba nanowires is characterized by a sign reversal of the spin component along the magnetic field and of the charge of bulk states with momenta close to $k=0$. Importantly, these findings are independent of the presence of MFs and rely only on bulk properties of the system. The boundary between phases is quite sharp but depends on the parameters of the system such as the SOI strength. These results open a way for mapping the phase diagram of the Rashba nanowire and bring a clear signature of the topological phase transition. Two types of experiments could be carried out to detect the spin or charge reversal at the topological phase transition point. The first one is based on spin-polarized STM spectroscopy which allows one to inject a current in the lowest bands [32-34,47]. Depending on the polarization of the STM probe, a current will flow or not in the trivial phase and the opposite situation will occur in the topological phase. The second possibility is to couple the nanowire to a quantum dot $[20,30,36,48,49]$, which then can be used for energy-selective spin read-out [50].

Acknowledgments. We acknowledge helpful discussions with Silas Hoffman, Marcel Serina, and Andreas Baumgartner. This work was support by the Swiss NSF and NCCR QSIT.
[1] M. Z. Hasan and C. L. Kane, Rev. Mod. Phys. 82, 3045 (2010).

[2] B. A. Bernevig and T. L. Hughes, Topological Insulators and Topological Superconductors (Princeton University Press, Princeton, NJ, 2013).

[3] A. Y. Kitaev, Ann. Phys. 303, 2 (2003).

[4] C. Nayak, S. H. Simon, A. Stern, M. Freedman, and S. D. Sarma, Rev. Mod. Phys. 80, 1083 (2008).

[5] L. Fu and C. L. Kane, Phys. Rev. Lett. 100, 096407 (2008).

[6] M. Sato and S. Fujimoto, Phys. Rev. B 79, 094504 (2009).

[7] R. M. Lutchyn, J. D. Sau, and S. D. Sarma, Phys. Rev. Lett. 105, 077001 (2010).

[8] Y. Oreg, G. Refael, and F. von Oppen, Phys. Rev. Lett. 105, 177002 (2010).

[9] J. Alicea, Phys. Rev. B 81, 125318 (2010).

[10] A. C. Potter and P. A. Lee, Phys. Rev. B 83, 094525 (2011).

[11] J. Klinovaja, S. Gangadharaiah, and D. Loss, Phys. Rev. Lett. 108, 196804 (2012).

[12] D. Chevallier, D. Sticlet, P. Simon, and C. Bena, Phys. Rev. B 85, 235307 (2012).

[13] J. Klinovaja, P. Stano, and D. Loss, Phys. Rev. Lett. 109, 236801 (2012).

[14] S. Nadj-Perge, I. K. Drozdov, B. A. Bernevig, and A. Yazdani, Phys. Rev. B 88, 020407(R) (2013).

[15] J. Klinovaja, P. Stano, A. Yazdani, and D. Loss, Phys. Rev. Lett. 111, 186805 (2013).
[16] B. Braunecker and P. Simon, Phys. Rev. Lett. 111, 147202 (2013).

[17] F. Maier, J. Klinovaja, and D. Loss, Phys. Rev. B 90, 195421 (2014).

[18] M. Thakurathi, A. A. Patel, D. Sen, and A. Dutta, Phys. Rev. B 88, 155133 (2013).

[19] F. Setiawan, K. Sengupta, I. B. Spielman, and J. D. Sau, Phys. Rev. Lett. 115, 190401 (2015).

[20] E. Vernek, P. H. Penteado, A. C. Seridonio, and J. C. Egues, Phys. Rev. B 89, 165314 (2014).

[21] G. L. Fatin, A. Matos-Abiague, B. Scharf, and I. Zutic, Phys. Rev. Lett. 117, 077002 (2016).

[22] J. Alicea, Rep. Prog. Phys. 75, 076501 (2012).

[23] C. W. J. Beenakker, Annu. Rev. Con. Mat. Phys. 4, 113 (2013).

[24] V. Mourik, K. Zuo, S. M. Frolov, S. R. Plissard, E. P. A. M. Bakkers, and L. P. Kouwenhoven, Science 336, 1003 (2012).

[25] H. O. H. Churchill, V. Fatemi, K. Grove-Rasmussen, M. T. Deng, P. Caroff, H. Q. Xu, and C. M. Marcus, Phys. Rev. B 87, 241401 (2013).

[26] A. Das, Y. Ronen, Y. Most, Y. Oreg, M. Heiblum, and H. Shtrikman, Nat. Phys. 8, 887 (2012).

[27] L. P. Rokhinson, X. Liu, and J. K. Furdyna, Nat. Phys. 8, 795 (2012).

[28] M. T. Deng, C. L. Yu, G. Y. Huang, M. Larsson, P. Caroff, and H. Q. Xu, Nano Lett. 12, 6414 (2012). 
[29] S. M. Albrecht, A. P. Higginbotham, M. Madsen, F. Kuemmeth, T. S. Jespersen, J. Nygard, P. Krogstrup, and C. M. Marcus, Nature (London) 531, 206 (2016).

[30] M. T. Deng, S. Vaitiekenas, E. B. Hansen, J. Danon M. Leijnse, K. Flensberg, J. Nygard, P. Krogstrup, and C. M. Marcus, Science 354, 6319 (2016).

[31] J. Chen, P. Yu, J. Stenger, M. Hocevar, D. Car, S. Plissard, E. P. A. M. Bakkers, T. Stanescu, and S. Frolov, arXiv:1610.04555

[32] S. Nadj-Perge, I. K. Drozdov, J. Li, H. Chen, S. Jeon, J. Seo, A. H. MacDonald, B. A. Bernevig, and A. Yazdani, Science 346, 602 (2014).

[33] M. Ruby, F. Pientka, Y. Peng, F. von Oppen, B. W. Heinrich, and K. J. Franke, Phys. Rev. Lett. 115, 197204 (2015).

[34] R. Pawlak, M. Kisiel, J. Klinovaja, T. Meier, S. Kawai, T. Glatzel, D. Loss, and E. Meyer, npj Quantum Inf. 2, 16035 (2016).

[35] E. J. H. Lee, X. Jiang, R. Aguado, G. Katsaros, C. M. Lieber, and S. D. Franceschi, Phys. Rev. Lett. 109, 186802 (2012).

[36] E. J. H. Lee, X. Jiang, M. Houzet, R. Aguado, C. M. Lieber, and S. D. Franceschi, Nat. Nanotechnol. 9, 79 (2014).

[37] J. Liu, A. C. Potter, K. T. Law, and P. A. Lee, Phys. Rev. Lett. 109, 267002 (2012)

[38] D. Bagrets and A. Altland, Phys. Rev. Lett. 109, 227005 (2012).

[39] D. I. Pikulin, J. P. Dahlhaus, M. Wimmer, H. Schomerus, and C. W. J. Beenakker, New J. Phys. 14, 125011 (2012).
[40] D. Rainis, L. Trifunovic, J. Klinovaja, and D. Loss, Phys. Rev. B 87, 024515 (2013).

[41] M. Thakurathi, O. Deb, and D. Sen, J. Phys.: Condens. Matter 27, 275702 (2015).

[42] T. D. Stanescu and S. D. Sarma, arXiv:1702.03976.

[43] C. R. Reeg and D. L. Maslov, Phys. Rev. B 95, 205439 (2017).

[44] See Supplemental Material at http://link.aps.org/supplemental/ 10.1103/PhysRevB.96.041401 for provide details on the wave functions and charge reversal.

[45] J. Klinovaja and D. Loss, Phys. Rev. B 86, 085408 (2012).

[46] J. Kammhuber, M. C. Cassidy, F. Pei, M. P. Nowak, A. Vuik, D. Car, S. R. Plissard, E. P. A. M. Bakkers, M. Wimmer, and L. P. Kouwenhoven, arXiv:1701.06878.

[47] D. Chevallier and J. Klinovaja, Phys. Rev. B 94, 035417 (2016).

[48] R. Chirla and C. P. Moca, Phys. Rev. B 94, 045405 (2016).

[49] D. B. Szombati, S. Nadj-Perge, D. Car, S. R. Plissard, E. P. A. M. Bakkers, and L. P. Kouwenhoven, Nat. Phys. 12, 568 (2016).

[50] R. Hanson, L. P. Kouwenhoven, J. R. Petta, S. Tarucha, and L. M. K. Vandersypen, Rev. Mod. Phys. 79, 1217 (2007).

[51] K. Björnson and A. M. Black-Schaffer, Phys. Rev. B 89, 134518 (2014).

[52] D. Sticlet, C. Bena, and P. Simon, Phys. Rev. Lett. 108, 096802 (2012). 